\title{
Laser interstitial thermal therapy for an eloquent region supratentorial brain lesion
}

\author{
*Mayur Sharma, MD,1 Daria Krivosheya, MD, FRCSC, ${ }^{2}$ Hamid Borghei-Razavi, MD, PhD, ${ }^{2}$ \\ Gene H. Barnett, MD, MBA, ${ }^{2}$ and Alireza M. Mohammadi, MD² \\ 'Department of Neurosurgery, University of Louisville, Kentucky; and ${ }^{2}$ Rose Ella Burkhardt Brain Tumor and Neuro-Oncology \\ Center, Department of Neurosurgery, Neurological Institute, Cleveland Clinic, Cleveland, Ohio \\ Laser interstitial thermal therapy (LITT) is a minimally invasive stereotactic technique that causes tumor ablation using \\ thermal energy. LITT has shown to be efficacious for the treatment of deep-seated brain lesions, including those near \\ eloquent areas. In this video, the authors present the case of a 62-year-old man with a history of metastatic melanoma \\ who presented with worsening right-sided hemiparesis. MRI revealed a contrast-enhancing lesion in left centrum \\ semiovale in close proximity to corticospinal tracts, consistent with radiation necrosis. The authors review their stepwise \\ technique of LITT with special attention to details for a lesion located near eloquent area.
}

The video can be found here: https://youtu.be/ndrTgi6MXqE.

KEYWORDS laser interstitial thermal therapy; LITT; eloquent; radiation necrosis; brain lesion 